\title{
Inventaire archéologique et habitat médiéval dans le canton de Lanta
}

Canton de Lanta

Myriam Daydé

\section{(2) OpenEdition \\ Journals}

Édition électronique

URL : http://journals.openedition.org/adlfi/10699

ISSN : 2114-0502

Éditeur

Ministère de la culture

\section{Référence électronique}

Myriam Daydé, «Inventaire archéologique et habitat médiéval dans le canton de Lanta », ADLFI.

Archéologie de la France - Informations [En ligne], Midi-Pyrénées, mis en ligne le 01 mars 1997, consulté le 03 mai 2019. URL : http://journals.openedition.org/adlfi/10699

Ce document a été généré automatiquement le 3 mai 2019.

(c) Ministère de la Culture et de la Communication, CNRS 


\title{
Inventaire archéologique et habitat médiéval dans le canton de Lanta
}

\author{
Canton de Lanta
}

Myriam Daydé

Date de l'opération : 1991 (PR)

Inventeur(s) : Daydé Myriam

1 Ce travail, sous le titre «Inventaire archéologique et habitat médiéval dans le canton de Lanta ", a été présenté comme mémoire de maîtrise à l'université de Toulouse - Le-Mirail.

2 Le canton de Lanta s'étend sur le Lauragais occidental. Situé à une vingtaine de kilomètres à l'est de Toulouse, il se compose de dix communes qui couvrent 10728 ha : Aigrefeuille, Aurin, Bourg-Saint-Bernard, Lanta, Lauzerville, Préserville, Saint-Pierre deLages, Sainte-Foy d'Aigrefeuille, Tarabel et Vallesville.

3 L'occupation gallo-romaine a été abordée afin d'en évaluer l'impact sur la formation des habitats médiévaux. La continuité entre les deux périodes (gallo-romaine et médiévale) s'avère plutôt faible. Un fort déséquilibre a en effet été observé entre le nombre de sites gallo-romains découverts et celui des sites médiévaux : trente-deux sites gallo-romains pour soixante sites médiévaux localisés. Parmi ces soixante sites, une quinzaine pourrait découler d'une implantation antique, du fait de leur relative proximité, de $100 \mathrm{~m}$ à $200 \mathrm{~m}$ environ (Roques, Sainte-Apollonie, le Pujol, etc.). Seuls cinq sites médiévaux sont implantés sur un site gallo-romain : le hameau des Peyrous et Saint-Martin de la Rivière à Tarabel, Sainte-Basilisse à Lanta, le hameau de Pied de Lanta au Bourg-Saint-Bernard, le site de Touron à Saint-Pierre-de-Lages et, enfin, certainement le village de Lauzerville.

Pour le Haut Moyen Âge, bien que certains sites, comme celui de Bonac ou de Capelle, aient fourni des vestiges anciens, il a été impossible d'en déterminer la période d'origine avec certitude. Seulement trois églises semblent dater de cette période : Saint-Anatoly, dont il est question dans le cartulaire de Saint-Sernin dès la fin du $\mathrm{X}^{\mathrm{e}} \mathrm{s}$., Sainte-Basilisse et Saint-Martin de Tarabel, qui sont bâties sur des sites gallo-romains. L'hagiotoponyme 
Saint-Sigismond, signalé au $\mathrm{XI}^{\mathrm{e}} \mathrm{s}$. au Pujol, pourrait également désigner une église du Haut Moyen Âge.

5 La plupart de ces églises se localisent sur des collines, mais il est intéressant de constater que certaines ont été bâties en plaine, ce qui ne se fera plus par la suite, hormis pour Sainte-Foy. De plus, sur quatre églises localisées dans la plaine, trois sont dédiées à SaintMartin et une à Saint-Pierre, vocables anciens et très populaires au Haut Moyen Âge. La localisation d'une église en plaine pourrait donc indiquer une origine très ancienne.

6 Sur onze sites ecclésiaux prospectés, huit églises ont attiré un habitat. Un seul, SaintMartin de la Rivière, pourrait avoir été fortifié, mais peut-être plus tardivement.

7 Pour le Moyen Âge, neuf mottes castrales ont été inventoriées avec certitude sur le canton, sept restant hypothétiques. Quatre sont encore existantes : Bellières à Sainte-Foy d'Aigrefeuille, Bugnac à Tarabel, la motte de la Dendelle à Saint-Pierre de Lages et la motte de Lanta. Cinq mottes ont disparu mais leur existence est bien attestée : Balause à Sainte-Foy d'Aigrefeuille, Bousquet, à Saint-Pierre de Lages, les Castillous à Aurin, la motte de Villèle à Préserville et celle de Saint-Paul à Bourg-Saint-Bernard. La majorité d'entre elles se situent dans le sud du canton (sept), laissant ainsi supposer que, dans le nord, les seigneurs y furent assez puissants pour en limiter le morcellement.

8 L'attraction de ces mottes sur l'habitat est variable mais lorsque tel fut le cas, l'habitat qui s'y est développé n'a en général pas subsisté au-delà du Moyen Âge. Seules les mottes de Lanta et des Castillous, ont engendré des communautés existant encore aujourd'hui.

9 Enfin, les sites de Préserville et deSaint-Paul comportaient, non pas une, mais deux mottes, ce qui peut être rapproché du site de Tarabel où la tradition orale rapporte également l'existence de deux buttes.

10 Le territoire cantonal totalise trois sites castraux: Lanta, Le Pujol et Tarabel, la fortification de Saint-Anatoly restant indéterminée. Ces trois castra possédaient des enceintes quadrangulaires. Lanta, et éventuellement Le Pujol, se sont développés à partir d'une motte castrale,ce qui n'est pas le cas du castrum de Tarabel. Le phénomène d'encastellement a provoqué, à Lanta et à Tarabel, une mutation des centres de peuplement, mais il semble que les toponymes précastraux aient résisté à ce changement puisque Tarabel et Lanta seraient des noms d'origine gauloise.

11 Quelques châteaux, qui ne semblent pas avoir été des châteaux érigés sur motte, ont probablement été construits vers la fin du Moyen Âge : Aurin, Calmontet, Charlus, La Garde et probablement Préserville et la Fraxinette. Ils sont tous bâtis en position dominante et ont engendré un habitat, du moins en ce qui concerne les quatre premiers sites. Cet habitat, souvent étendu, prouve que ces châteaux ont eu une certaine influence sur le territoire environnant. Pourtant, seulement deux communautés, sur les quatre premiers sites mentionnés, ont perduré jusqu'à nos jours: le hameau de La Garde et Aurin. Durant le Moyen Âge, neuf églises semblent avoir été édifiées. Hormis l'église de SainteFoy, toutes sont érigées sur des hauteurs. Seulement trois d'entre elles ont développé un habitat : Sainte-Foy et Sainte-Apollonie. L'habitat d'origine ecclésiale apparait restreint.

13 Les documents du Bas Moyen Âge mentionnent souvent l'existence de hameaux, qui ont parfois été localisés avec exactitude grâce à la prospection. Celle-ci a également permis d'en découvrir de nouveaux, qui quadrillent tout le Lantarès et indiquent une forte population. Certains découleraient de sites gallo-romains, d'autres sont vraisemblablement apparus autour d'oratoires ou de chapelles: Saint-Jean-de-la-Selve, 
les Roujols, etc., ou même de fortifications. Mais le plus souvent, leur origine reste obscure : Agasselle, Cosirou, Cunq, Garnès, etc.

14 Le Bas Moyen Âge est, semble-t-il, une période stérile en matière d'habitat. Seules quelques fortifications ont été construites sur le territoire cantonal. Le type le plus répandu est la maison forte : Salle du Pech, Le Ferradou, Barthecave et Foucaud. Elles ne se localisent que dans le sud du canton et n'ont pas, excepté la Salle du Pech qui est sans doute la plus ancienne, attiré d'habitat. Bourg-Saint-Bernard, malgré l'étymologie de son nom, serait une bastide fortifiée qui aurait été bâtie au début du XIII ${ }^{\mathrm{e}} \mathrm{s}$.

INDEX

Index chronologique : bas Moyen Âge, haut Moyen Âge, Moyen Âge operation Prospection (PR) 